\title{
18F-FDG PET/MRI in adult sarcomas
}

\author{
Gianluca Cassarino $^{1} \cdot$ Laura Evangelista $^{1} \cdot$ Chiara Giraudo $^{2} \cdot$ Alfio Capizzi $^{3} \cdot$ Giovanni Carretta $^{3}$ - Pietro Zucchetta ${ }^{1}$. \\ Diego Cecchin ${ }^{1}$ (1)
}

Received: 31 July 2020 / Accepted: 29 September 2020 / Published online: 20 October 2020

(c) The Author(s) 2020

\begin{abstract}
Aim This mini-review aims to summarize the main findings on PET/MRI in patients with sarcomas.

Materials and methods A literature search was carried out on MEDLINE databases, such as PubMed, Scopus, Web of Science and Google Scholar, using the search terms "PET", "positron emission tomography", "PET/MRI", "positron emission tomography/magnetic resonance imaging", and "sarcoma". No restrictions (i.e. language, age, type of manuscript, or the like) were applied to the search strategy. Abstracts, reviews, letters to editors, and editorials were excluded.

Results Eight studies were ultimately included. From 2013 to 2019, a total of 154 adult patients with sarcomas underwent 18F-fluorodeoxyglucose (18F-FDG) PET/MRI. Of these patients, 129 had soft tissue sarcomas (STS), 5 had bone sarcomas, and 20 had cardiac masses. Thirty-two patients had PET/MRI for early-stage disease, 45 for recurrences, and 57 for the assessment of response to treatment. For staging purposes, the studies suggest that PET/MRI can significantly improve the delineation of surgical margins. At restaging, PET/MRI can also detect sarcoma recurrences more accurately than conventional imaging.

Conclusions 18F-FDG PET/MRI has promising indications in patients with sarcomas, from disease staging to the assessment of response to treatment. Further studies are warranted to confirm these results, especially in subgroups with specific histopathological features.
\end{abstract}

Keywords Positron emission tomography $\cdot$ Magnetic resonance imaging $\cdot$ Sarcomas $\cdot 18$ F-FDG $\cdot$ Cancer staging

\section{Introduction}

Sarcomas are malignant tumors of connective tissue that account for less than $1 \%$ of all malignant cancers $[1,2]$. Soft tissue sarcomas (STS) develop in muscle, joints, blood vessels and subcutis, and are relatively frequent in adults,

Electronic supplementary material The online version of this article (https://doi.org/10.1007/s40336-020-00395-9) contains supplementary material, which is available to authorized users.

Diego Cecchin

diego.cecchin@unipd.it

1 Nuclear Medicine Unit, Department of Medicine (DIMED), University-Hospital of Padova, Via Giustiniani 2, 35128 Padua, Italy

2 Radiology Unit, Department of Medicine (DIMED), University-Hospital of Padova, Padua, Italy

3 Department of Directional Hospital Management, University-Hospital of Padova, Padua, Italy whereas bone sarcomas typically affect a younger population [3].

The diagnosis of sarcoma is based mainly on clinical examination, ultrasound and/or magnetic resonance imaging (MRI), and biopsy. MRI is highly sensitive in the tumor's detection and local staging. It can reveal the heterogeneous features of sarcomas (such as the presence of necrosis, or adipose or fibrous tissue) and their relation to neighboring structures. MRI should always be performed prior to surgery to have a better chance of achieving resections with clear margins while sparing adjacent structures and avoiding loss of function.

Computed tomography (CT) is often used to identify pulmonary and visceral metastases. Positron emission tomography (PET) combined with CT and using 18F-fluorodeoxyglucose (18F-FDG) has demonstrated a high diagnostic accuracy in the identification of distant metastases at staging [4] and restaging [5].

A simultaneous PET/MRI acquisition can combine the highly sensitive molecular imaging of 18F-FDG PET with 
the superior soft-tissue contrast of MRI scans [6, 7]. This mini-review aims to summarize the main findings on PET/ MRI in patients with sarcomas.

\section{Materials and methods}

Three reviewers (G.C., L.E., D.C.) conducted the literature search, study inclusion, and data extraction procedures. A literature search for studies on "PET", "positron emission tomography", "PET/MRI", "positron emission tomography/ magnetic resonance imaging" and "sarcoma" was run on MEDLINE databases (PubMed, Scopus, Web of Science and Google Scholar). No limits (i.e. language, age, type of manuscript, or the like) were applied to the search strategy. Number of enrolled patients was not considered as an exclusion criterion, while abstracts, reviews, letters to editors, and editorials were not included. The search was extended to articles referenced in the literature identified to find any additional reports meeting the inclusion criteria. Data on outcomes were retrieved from each article. Quality assessment of the included studies was performed (Table 1 supplemental material) using the Quality Assessment of Diagnostic Accuracy Studies tool-2 (QUADAS-2) [8].

\section{Results}

Figure 1 shows the flowchart adopted for the literature search. Eight studies were selected for the purposes of the present contribution. Table 1 summarizes the characteristics of the selected reports [6, 9-15].

Three papers $[6,9,10]$ showed a high risk of bias for the patient selection and unclear risk for the flow and timing. Moreover, the papers by Schuler et al. [9] and Partovi et al. [10] demonstrated a high risk of bias for the reference standard. Similarly, the applicability concerns for the patient selection was high in three reports $[6,9,10]$. The results of QUADAS-2 analysis are illustrated in Table 1s.

From 2013 to 2019, a total of 154 patients with sarcomas underwent 18F-FDG PET/MRI. There were 129 cases of soft tissue sarcoma (STS), 5 bone sarcomas, and 20 patients had cardiac sarcomas. Thirty-two patients had PET/MRI for early-stage disease, 45 for recurrences, and 57 for the assessment of response to treatment.
Fig. 1 PRISMA flowchart for the literature search. From: Moher D, Liberati A, Tetzlaff J, Altman DG, The PRISMA Group (2009) Preferred Reporting Items for Systematic Reviews and Meta-Analyses: The PRISMA Statement. PLoS Med 6(6): e1000097. doi: https://doi.org/10.1371/journ al.pmed1000097. For more information, visit https://www. prisma-statement.org
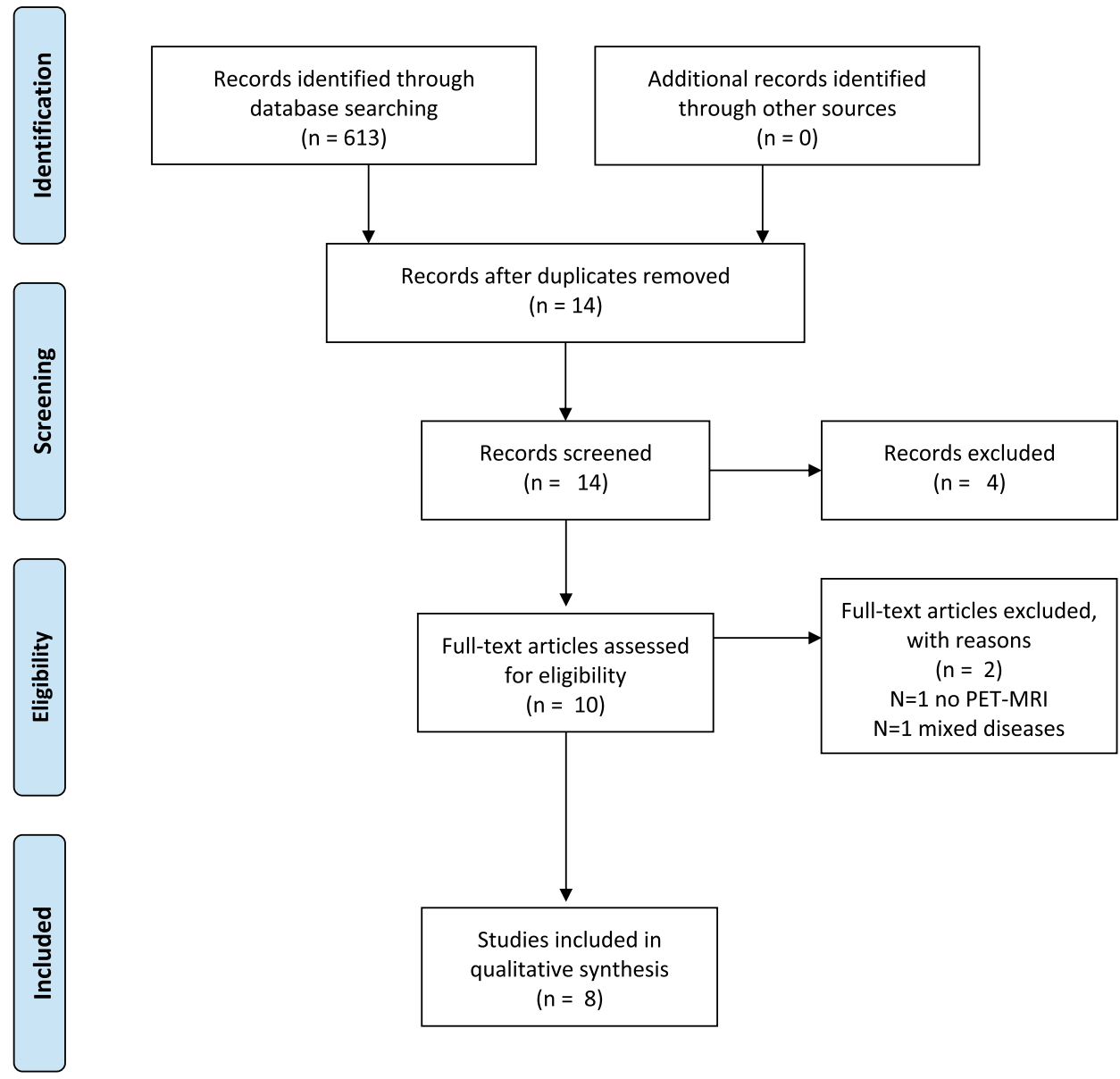


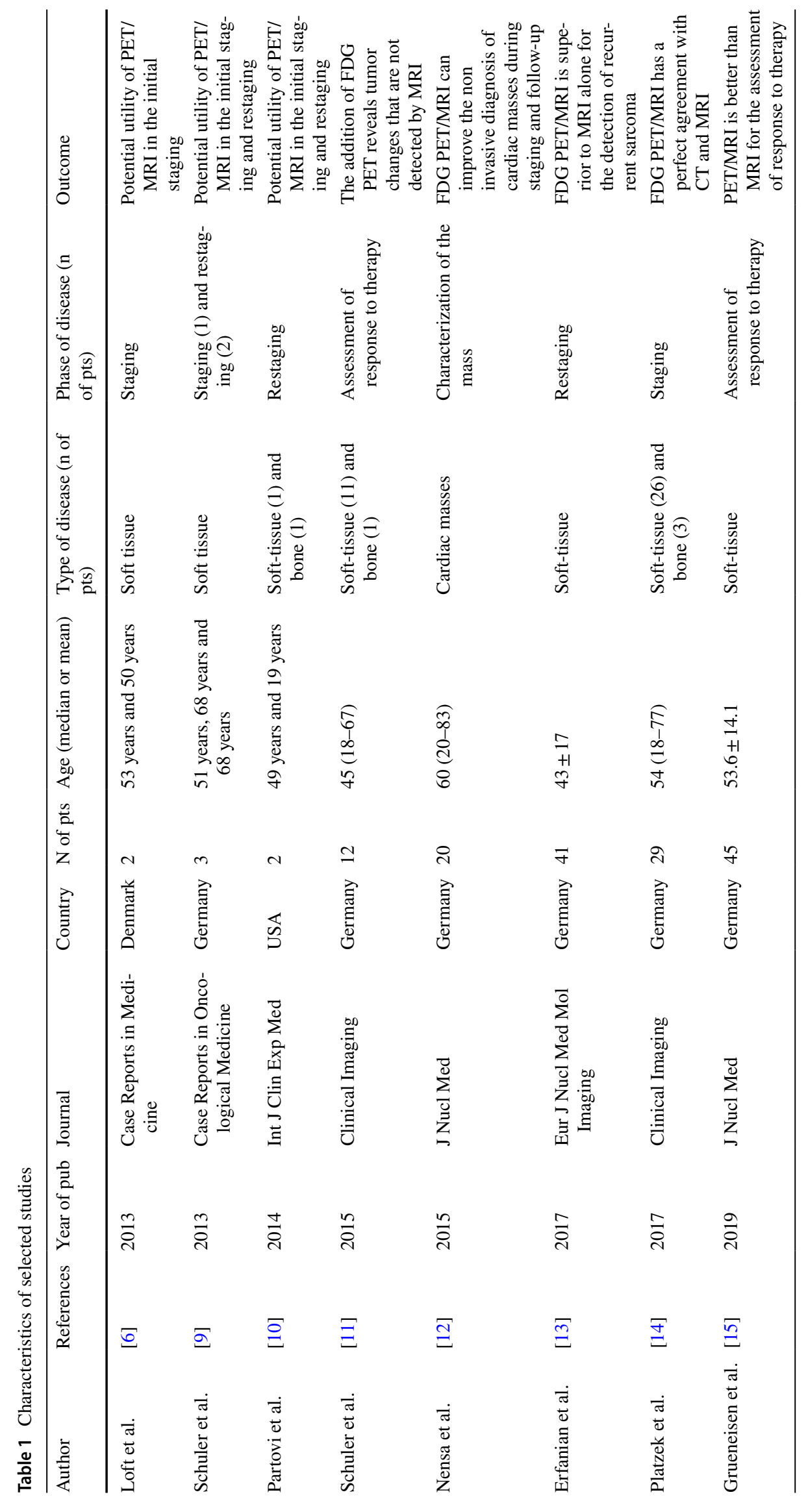


The diagnostic performances in terms of sensitivity, specificity, positive predictive value, negative predictive value and accuracy of PET/MRI for the different settings of the disease have been reported in Table 2.

\section{PET/MRI for initial disease staging}

Loft et al. [6] reported that it was only in 2 patients that PET/ MRI provided valuable information about possible tumor invasion of adjacent bone and nerves significantly improving the delineation of the surgical margins. It was suggested that the main advantage of simultaneous PET and MRI images lies in a more limited variability in signal intensity and illdefined margins.

Schuler et al. [9] assessed the role of PET/MRI before starting neoadjuvant therapy in a 51-year-old man affected by a rhabdomyosarcoma of the proximal thigh, suspicious for distant metastases at CT images. A high SUVmax was reported in the primary lesion, while a slight uptake of FDG was depicted in the inguinal lymph nodes. Therefore, the patient was sent to neoadjuvant therapy.

Nensa et al. [12] evaluated the role of PET/MRI in 20 patients with cardiac masses. The histological evaluation was available in 15 patients, while additional imaging examinations (such as CT and MRI) were used in the residual 5 patients. In accordance with the standard of references, 12 cardiac masses were defined as benign and 8 as malignant. The authors underlined that the association between a SUVmax cut-off value of 5.2 and all available MRI features (i.e. tumor volume, T2w hyperintensity, contrast enhancement, pericardial effusion and morphological features at cine-MRI) reached a sensitivity and a specificity of $100 \%$.

Platzek et al. [14] reported that PET/MRI provided similar information as compared to MRI and CT in patients with sarcoma. They enrolled 29 patients, 26 with STS and 3 with bone sarcomas. PET/MRI proved slightly more sensitive than conventional imaging in identifying distant metastases (97.8\% vs. $94.4 \%$, respectively). The authors reported that PET/MRI offers some advantages in the initial staging of disease because it can add prognostic information and be useful for monitoring response to therapy.

Figures 2 and 3 show two examples of 18F-FDG PET/ MRI acquired with Biograph Siemens mMR at Padova University Hospital for staging purposes in patients with a proven diagnosis of STS.

\section{PET/MRI for restaging}

Schuler et al. [9], in 2 patients with recurrent sarcomas demonstrated that PET/MRI was able to early recognize the presence of recurrence and, therefore, to guide an appropriate treatment (systemic chemotherapy in both the cases). Similarly, Partovi et al. [10] showed that the addiction of PET to MRI information was able to clarify the malignant nature of a psoas mass in one patient previously treated with surgery and radiotherapy. The same authors stated that PET/MRI was able, as a one-stop-shop study, to stage the recurrence of soft-tissue sarcomas in a 19-years old female, identifying both bone marrow and bone lytic lesions.

Based on data published by Erfanian et al. [13], PET/ MRI can detect the recurrence of high-grade sarcoma more

Table 2 Diagnostic performances of the selected articles

\begin{tabular}{|c|c|c|c|c|c|c|c|c|}
\hline Author & References & Setting of disease & Imaging technique & Sensitivity & Specificity & PPV & NPV & Accuracy \\
\hline Loft et al. & [6] & Staging & PET/MRI & NA & NA & NA & NA & NA \\
\hline Schuler et al. & [9] & Staging and restaging & PET/MRI & NA & NA & NA & NA & NA \\
\hline Partovi et al. & {$[10]$} & Restaging & PET/MRI & NA & NA & NA & NA & NA \\
\hline Schuler et al. & [11] & $\begin{array}{l}\text { Assessment of response to } \\
\text { therapy }\end{array}$ & PET/MRI & $100 \%$ & - & $100 \%$ & - & $100 \%$ \\
\hline Nensa et al. & [12] & Characterization of the mass & $\begin{array}{l}\text { SUVmax cut-off } 5.2 \\
\text { All MRI features } \\
\text { MRI and SUVmax } 5.2\end{array}$ & $\begin{array}{l}100 \% \\
100 \% \\
100 \%\end{array}$ & $\begin{array}{l}92 \% \\
92 \% \\
100 \%\end{array}$ & $\begin{array}{l}- \\
- \\
-\end{array}$ & $\begin{array}{l}- \\
- \\
-\end{array}$ & $\begin{array}{l}- \\
- \\
-\end{array}$ \\
\hline Erfanian et al. & {$[13]$} & Restaging & $\begin{array}{l}\text { PET/MRI (p-b) } \\
\text { PET/MRI (1-b) } \\
\text { MRI alone (p-b) } \\
\text { MRI alone (l-b) }\end{array}$ & $\begin{array}{l}96.3 \% \\
95 \% \\
81.5 \% \\
80 \%\end{array}$ & $\begin{array}{l}78.6 \% \\
76.5 \% \\
85.7 \% \\
82.4 \%\end{array}$ & $\begin{array}{l}89.7 \% \\
90.5 \% \\
91.7 \% \\
91.4 \%\end{array}$ & $\begin{array}{l}91.7 \% \\
86.7 \% \\
70.6 \% \\
63.6 \%\end{array}$ & $\begin{array}{l}90 \% \\
89.5 \% \\
82.9 \% \\
80.7 \%\end{array}$ \\
\hline Platzek et al. & [14] & Staging & $\begin{array}{l}\text { PET/MRI (M stage) } \\
\text { Conventional Imaging (M } \\
\text { stage) }\end{array}$ & $\begin{array}{l}97.8 \% \\
94.4 \%\end{array}$ & $\begin{array}{l}100 \% \\
100 \%\end{array}$ & - & - & $\begin{array}{l}- \\
-\end{array}$ \\
\hline Grueneisen et al. & {$[15]$} & $\begin{array}{l}\text { Assessment of response to } \\
\text { therapy }\end{array}$ & PET/MRI & NA & NA & NA & NA & NA \\
\hline
\end{tabular}

$P P V$ positive predictive value, $N P V$ negative predictive value, $N A$ not available, $S U V$ max maximum standardized uptake value, $p$ - $b$ patientbased, $l-b$ lesion-based, $M$ metastatic 


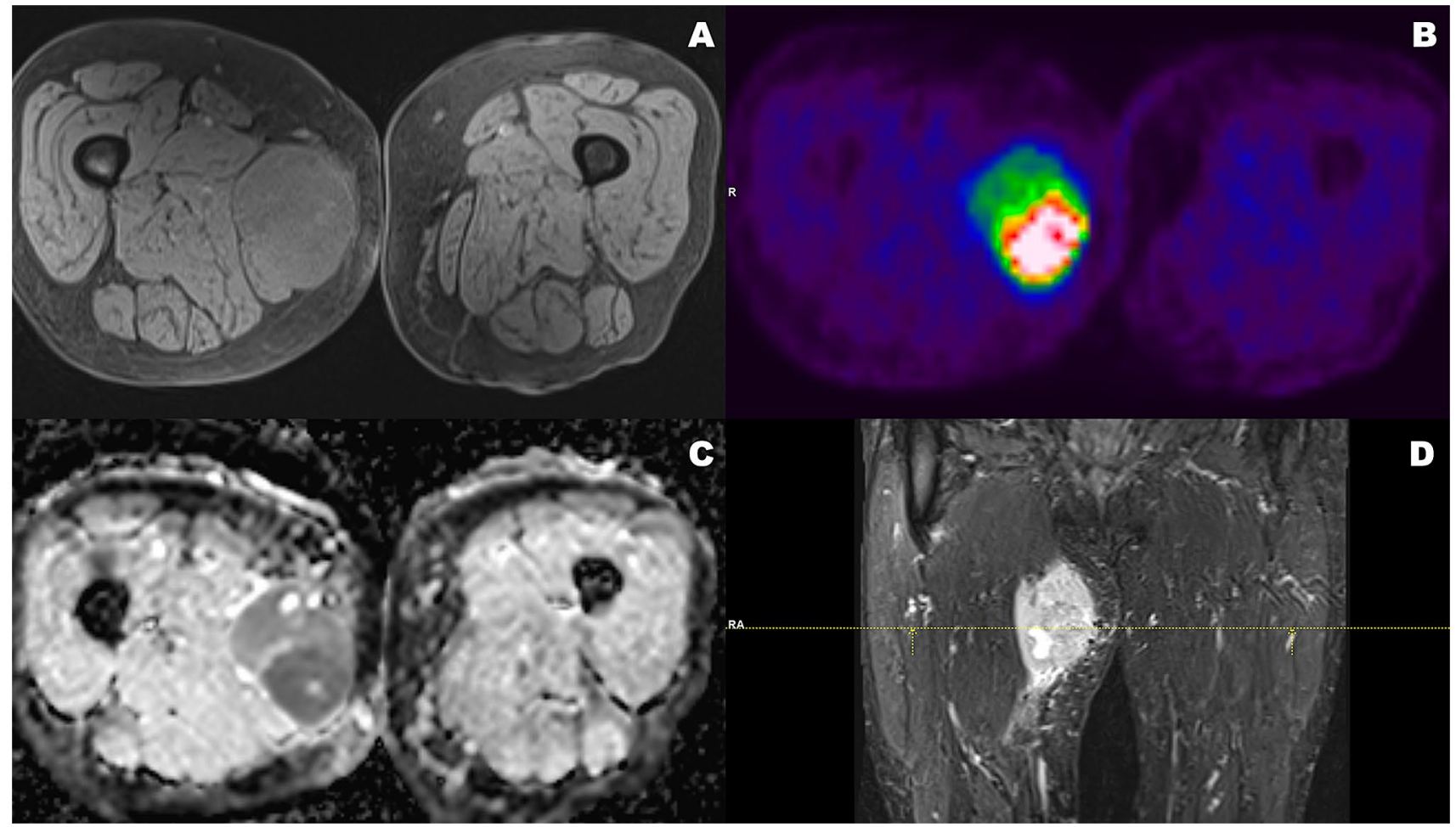

Fig. 2 A female with a high-grade pleomorphic fibrosarcoma of the right thigh (a: axial T1 VIBE). Baseline PET/MRI demonstrated an inhomogeneous 18F-FDG uptake in the main lesion (b: axial 18FFDG PET), with a high uptake in the posterior area, and a low uptake in the anterior region (compatible with necrosis). MRI showed a slight invasion of surrounding muscles (d: coronal T1 STIR), and an inhomogeneous DWI signal (c: axial DWI ADC map). The patient underwent radical excision followed by adjuvant radiotherapy

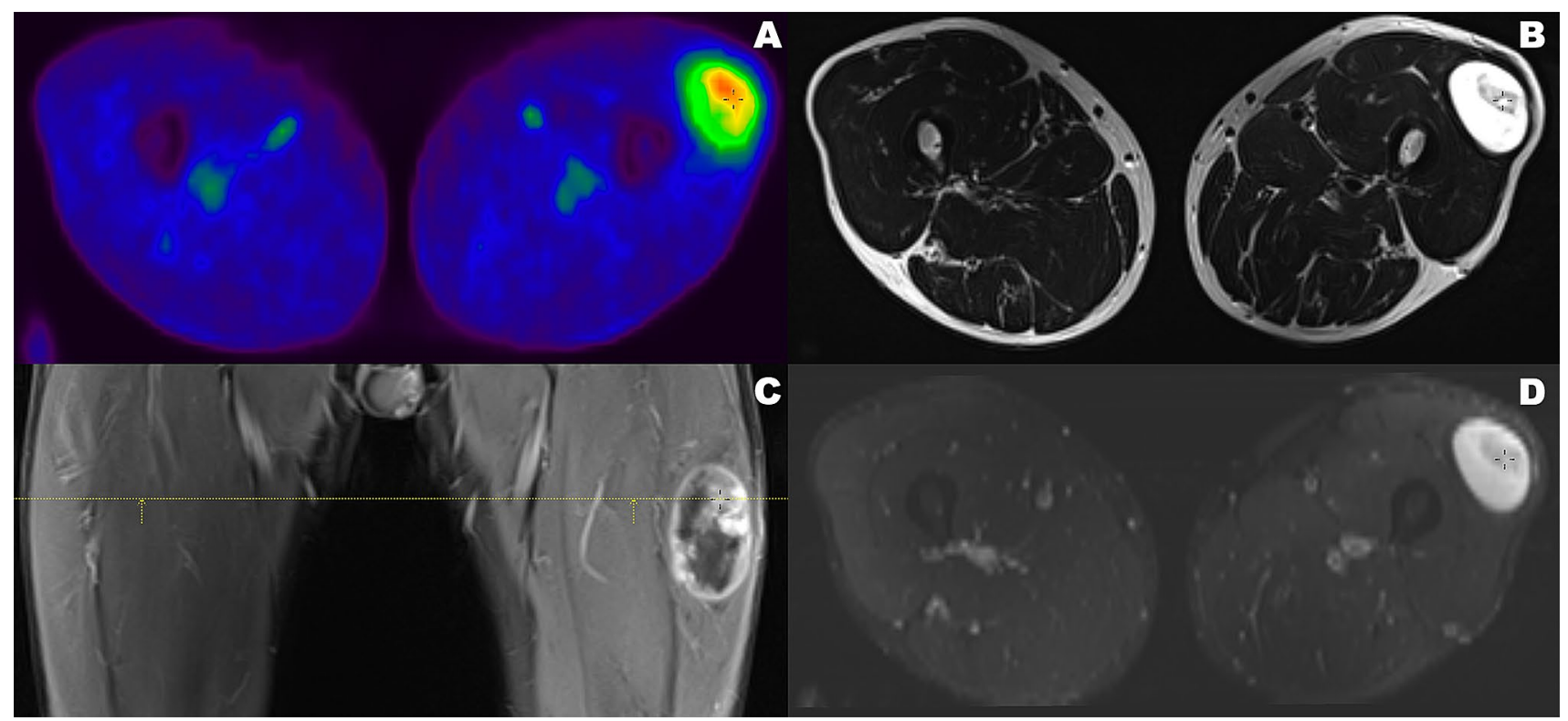

Fig. 3 A male with a high-grade myxofibrosarcoma of the left thigh. PET/MRI performed for initial staging showed a low 18F-FDG uptake (a: axial 18 F-FDG) in a large mass $(6.5 \times 4.5 \mathrm{~cm})$ with per- ilesional edema (b: axial T2-TSE; c: coronal T1 VIBE after contrast medium d: axial T1 STIR). The patient was treated with neoadjuvant radiotherapy and surgery 
accurately than MRI alone, in both patient- and lesion-based analyses $(96.3 \%$ vs. $81.4 \%$, and $95 \%$ vs. $80 \%$, respectively). Non-specific $18 \mathrm{~F}-\mathrm{FDG}$ accumulation in post-therapy fibrotic tissue can increase the number of false-positive findings albeit without affecting the specificity.

Figure 4 shows an image from a patient with recurrent STS acquired using Biograph Siemens mMR at Padova University Hospital.

\section{PET/MRI for the assessment of response to therapy}

Schuler et al. [11] studied 12 patients who underwent FDG PET/MRI before and after neoadjuvant chemotherapy. For the evaluation of response to therapy, EORTC (European Organization for Research and Treatment) and Choi criteria were used, respectively, for PET and MR images. Based on the histological analysis, available in 7 out of 12 patients, 6/7 (86\%) patients were correctly identified according to Choi criteria, whereas 4/7 (57\%) would have been correctly identified by EORTC criteria.

Some years later, Grueneinsen et al. [15] reported that the PERCIST (Positron Emission Tomography Response Criteria In Solid Tumors 1.0) performed better than the RECIST (Response Evaluation Criteria in Solid Tumors), or the Choi criteria for the purpose of assessing response to therapy in 45 sarcoma patients treated with isolated limb perfusion.

\section{Discussion and conclusions}

In the present mini-review, the majority of papers presents data about soft tissue sarcomas that represent the commonest form of sarcoma in the adults, while malignant bone tumors are about $10 \%$ of sarcomas [1]. Furthermore, cardiac sarcomatous masses described in literature are very rare but could be probably better assessed using PET/MRI rather than PET/ $\mathrm{CT}$ as reported by Nensa et al. [12].

As emerges from the available evidence of PET/MRI used for STS and bone sarcomas, hybrid imaging seems to provide some additional information in the preoperative setting: being able to disclose the real local extent of disease, its use can prevent the risk of overtreatment. Tumor margins are often affected by inflammation and edema, and PET/MRI delineated them more accurately than contrast-enhanced MRI alone [6]. A more precise and specific image, such as the one generated by PET/MRI, may, therefore, reduce morbidity due to overtreatment (e.g. unnecessary amputations). MRI is still considered the reference standard in the preoperative phase, however, due to the limited availability of
Fig. 4 A male with low-grade myxofibroma of the left tibia treated with multiple excisions from 2010 to 2014, and external radiotherapy in 2011. In 2015, he was referred for $18 \mathrm{~F}-\mathrm{FDG}$ PET/MRI for a suspected distant recurrence. The images showed a significant $18 \mathrm{~F}-\mathrm{FDG}$ uptake in the soma of D12 (red arrow) associated with a collapse of the vertebra compatible with disease recurrence. The patient was treated with vertebroplasty and radiotherapy
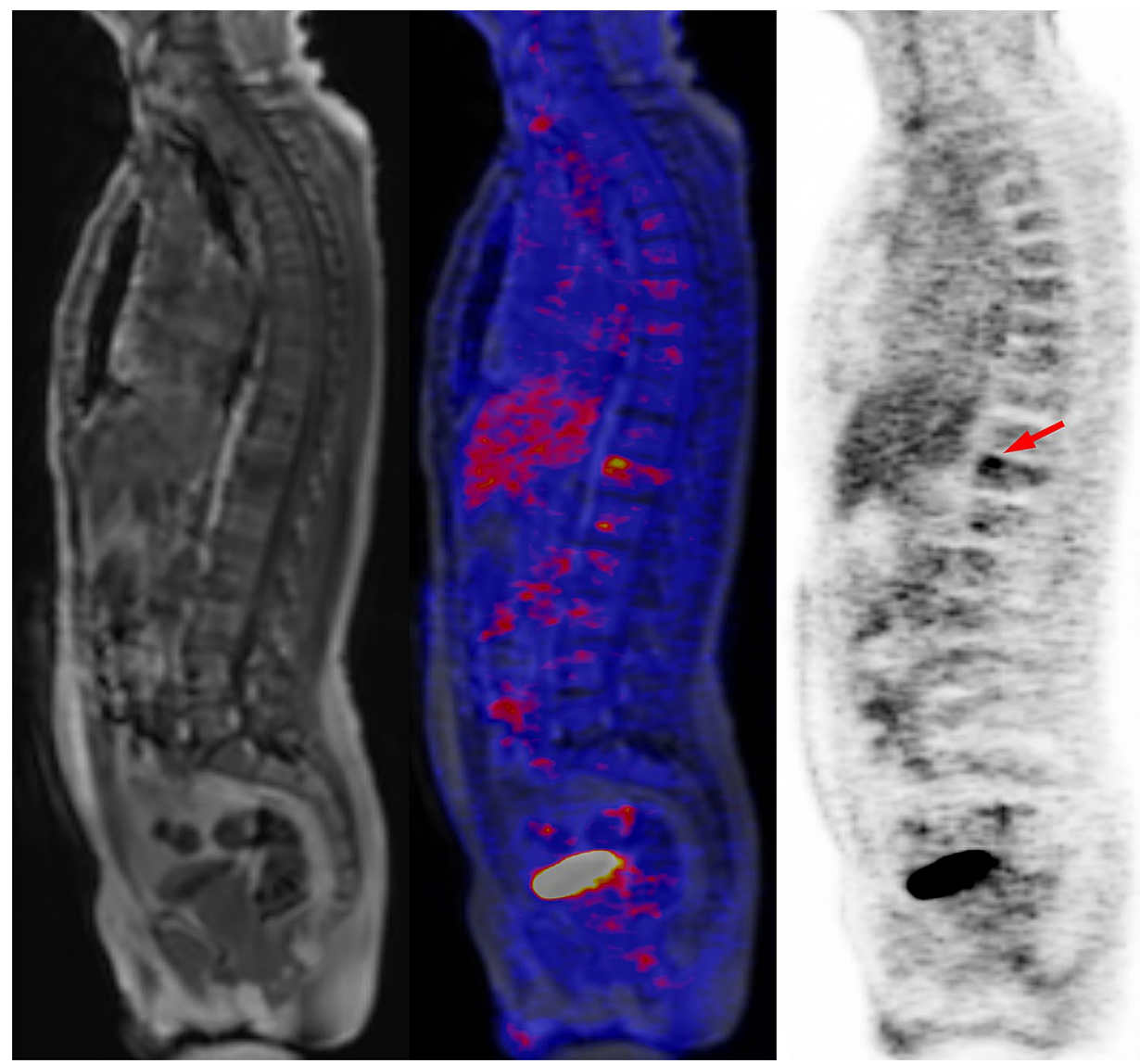
Table 3 Technical characteristics of the studies

\begin{tabular}{|c|c|c|c|c|c|}
\hline Authors & Ref & Type of scanner & FDG dosage & MRI protocol & PET protocol \\
\hline Loft et al. & {$[6]$} & Biograph mMR, Siemens & $4 \mathrm{MB} / \mathrm{kg}$ & $\begin{array}{l}\text { DIXON (AC), STIR, T1-weighted TSE, axial T2-fast SE } \\
\text { (with c.e) }\end{array}$ & $5 \mathrm{~min} / \mathrm{bed}$ \\
\hline Schuler et al. & [9] & Ingenuity PET/MRI, Philips & NA & NA & NA \\
\hline Partowi et al. & [10] & NA & NA & NA & NA \\
\hline Schuler et al. & [11] & Ingenuity PET/MRI, Philips & 4.5 MBq/Kg & T1 gradient-echo (AC) (with c.e) & $2 \mathrm{~min} / \mathrm{bed}$ \\
\hline Nensa et al. & [12] & Biograph mMR, Siemens & $199 \pm 58 \mathrm{MBq}$ & $\begin{array}{l}\text { DIXON (AC), HAST, Turbo-SPIN ECHO, T1, T2 (with } \\
\text { c.e) }\end{array}$ & NA \\
\hline Erfanian et al. & [13] & Biograph mMR, Siemens & $4 \mathrm{MB} / \mathrm{Kg}$ & $\begin{array}{l}\text { DIXON (AC), STIR, T1-weighted TSE, axial T2-fast } \\
\text { TSE, DWI, T1-weighted VIBE, T1w FLASH, } \\
\text { T1-weighted VIBE (with c.e) }\end{array}$ & $10 \mathrm{~min} / \mathrm{bed}$ \\
\hline Platzek et al. & [14] & Biograph mMR, Siemens & 4.5 MBq/Kg & T1 gradient-echo (AC), T1-weighted TSE (with c.e) & NA \\
\hline Grueneisen et al. & [15] & Biograph mMR, Siemens & $247 \pm 48 \mathrm{MBq}$ & T1-TSE, T2-TSE and VIBE (with c.e) & $10 \mathrm{~min} / \mathrm{bed}$ \\
\hline
\end{tabular}

$N A$ not available, $A C$ attenuation correction

PET/MRI scanners, and the lack of large-scale multicenter studies on the issue.

As shown in Fig. 2, PET images can identify the different glucose metabolism in different areas of a large tumor with similar MRI characteristics. The concomitant metabolic and morphological information provided by PET/MRI can be helpful in orienting tumor biopsy procedures, achieving a significant reduction in the burden of radiation by comparison with PET/CT $[16,17]$. This should be considered a very important factor when dealing with younger patients.

When applied to recurrent disease, PET/MRI affords a more accurate differentiation between necrosis and hematoma because it has a higher soft-tissue resolution than PET/ CT [10]. It can also add information not provided by MRI or CT alone (in the case of adenopathy, for instance, or doubtful sites of disease). Figure 4 illustrates our experience with a patient who had an unclear skeletal recurrence on MRI that PET images were able to confirm. The main limitation of PET/MRI, on the other hand, seems to lie in the detection of lung metastases [18]. A CT of the thorax delivers valuable information with regard to lung metastases, while MRI seems not yet suitable for this setting.

The combination of surrogate markers obtainable with PET and MRI can be useful for assessing response to therapy in sarcoma patients [10, 19]. 18F-FDG PET can disclose early metabolic changes in a tumor, while MRI-using particular sequences, i.e. diffusion-weighted imaging (DWI) with apparent diffusion coefficient (ADC) maps-can help distinguish viable tumors from fibrosis or necrosis. The differentiation between tumor and necrosis is of particular interest for restaging purposes after radiation therapy or chemotherapy and for prognosis [10].

Quantitative data provided by PET/MRI and MRI alone were available only in 2 studies. Erfanian et al. [13] reported that, in patients with suspicious recurrence of sarcoma, the malignant lesions have a higher median
SUVmax than the benign ones (6.7 vs. 2.1) and also the volume assessed by MRI alone was higher in the malignant subset than the counterpart (34.7 vs. $24 \mathrm{~mm}$ ). The study by Nensa et al. [12] showed that the SUVmax of 5.2 was able to discriminate between benign vs. malignant cardiac masses with a high diagnostic performance. Data from PET/CT showed that similar results can be obtained using a SUVmax cut-off value of 4.6 [20]. Differences of SUVmax cut-offs between PET/MRI and PET/CT can be due to the different approaches for attenuation correction [21].

Some limitations are present in the selected papers and, therefore, in the present mini-review. First, the low number of enrolled patients. Second, different settings of the disease have been considered thus including only few patients for each group. Third, the histological characteristics of primary and recurrent tumors are different. However, as largely reported, sarcoma is heterogenous with various subtypes having a different prognostic impact [22]. This latter aspect is correlated with a different tumor metabolic behaviour. Finally, different PET/MRI protocol acquisition (see Table 3) have been used. An harmonization of PET/MRI protocol to be used for sarcomas should be, to us, encouraged to improve future multicentric studies.

\section{Future perspectives}

In conclusion, 18F-FDG PET/MRI has promising indications in patients with sarcomas especially for early staging and assessing response to treatment. The value of this hybrid imaging modality should be further explored in harmonized multicentric studies, using both conventional and novel radiopharmaceutical agents (such as for example 18F-FAZA, 64Cu-ATSM [23] and 18F-FAPI [24]). 
Funding Open access funding provided by Università degli Studi di Padova within the CRUI-CARE Agreement.

\section{Compliance with ethical standard}

Conflict of interest Gianluca Cassarino: nothing to disclose. Laura Evangelista: nothing to disclose. Chiara Giraudo: nothing to disclose. Alfio Capizzi: nothing to disclose. Giovanni Carretta: nothing to disclose. Pietro Zucchetta: nothing to disclose. Cecchin Diego: nothing to disclose.

Research involving human participants and/or animals The present manuscript does not involve human participants.

Informed consent The present manuscript does not require an informed consent collection.

Open Access This article is licensed under a Creative Commons Attribution 4.0 International License, which permits use, sharing, adaptation, distribution and reproduction in any medium or format, as long as you give appropriate credit to the original author(s) and the source, provide a link to the Creative Commons licence, and indicate if changes were made. The images or other third party material in this article are included in the article's Creative Commons licence, unless indicated otherwise in a credit line to the material. If material is not included in the article's Creative Commons licence and your intended use is not permitted by statutory regulation or exceeds the permitted use, you will need to obtain permission directly from the copyright holder. To view a copy of this licence, visit http://creativecommons.org/licenses/by/4.0/.

\section{References}

1. Burningham Z, Hashibe M, Spector L, Schiffman JD (2012) The epidemiology of sarcoma. Clin Sarcoma Res 2(1):14

2. Siegel RL, Miller KD, Jemal A (2015) Cancer statistics. CA A Cancer J Clin 65(1):5

3. Bauer HC, Trovik CS, Alvegård TA, Berlin O, Erlanson M, Gustafson P, Klepp R, Möller TR, Rydholm A, Saeter G, Wahlström O, Wiklund T (2001) Monitoring referral and treatment in soft tissue sarcoma: study based on 1,851 patients from the scandinavian sarcoma group register. Acta Orthop Scand 72(2):150-159

4. Macpherson RE, Pratap S, Tyrrell H et al (2018) Retrospective audit of 957 consecutive 18F-FDG PET-CT scans compared to CT and MRI in 493 patients with different histological subtypes of bone and soft tissue sarcoma. Clin Sarcoma Res. 8:9

5. Charest M, Hickeson M, Lisbona R, Novales-Diaz JA, Derbekyan V, Turcotte RE (2009) FDG PET/CT imaging in primary osseous and soft tissue sarcomas: a retrospective review of 212 cases. Eur J Nucl Med Mol Imaging 36(12):1944-1951

6. Loft A, Jensen KE, Löfgren J, Daugaard S, Petersen MM (2013) PET/MRI for preoperative planning in patients with soft tissue sarcoma: a technical report of two patients. Case Rep Med 2013:791078

7. Ratib O, Beyer T (2011) Whole-body hybrid PET/MRI: ready for clinical use? Eur J Nucl Med Mol Imaging 38(6):992-995

8. Whiting PF, Rutjes AW, Westwood ME et al (2011) QUADAS-2: a revised tool for the quality assessment of diagnostic accuracy studies. Ann Intern Med 155(8):529-536
9. Schuler MK, Richter S, Beuthien-Baumann B, Platzek I, Kotzerke J, van den Hoff J, Ehninger G, Reichardt P (2013) PET/MRI imaging in high-risk sarcoma: first findings and solving clinical problems. Case Rep Oncol Med 2013:793927

10. Partovi S, Kohan AA, Zipp L et al (2014) Hybrid PET/MR imaging in two sarcoma patients-clinical benefits and implications for future trials. Int J Clin Exp Med 7(3):640-648

11. Schuler MK, Platzek I, Beuthien-Baumann B, Fenchel M, Ehninger G, van den Hoff J (2015) (18)F-FDG PET/MRI for therapy response assessment in sarcoma: comparison of PET and MR imaging results. Clin Imaging 39(5):866-870

12. Nensa F, Poeppel T, Tezgah E et al (2015) Integrated FDG PET/ MR imaging for the assessment of myocardial salvage in reperfused acute myocardial infarction. Radiology 276(2):400-407

13. Erfanian Y, Grueneisen J, Kirchner J et al (2017) Integrated 18FFDG PET/MRI compared to MRI alone for identification of local recurrences of soft tissue sarcomas: a comparison trial. Eur J Nucl Med Mol Imaging 44(11):1823-1831

14. Platzek I, Beuthien-Baumann B, Schramm G et al (2017) FDG PET/MR in initial staging of sarcoma: Initial experience and comparison with conventional imaging. Clin Imaging 42:126-132

15. Grueneisen J, Schaarschmidt B, Demircioglu A et al (2019) 18F-FDG PET/MRI for therapy response assessment of isolated limb perfusion in patients with soft-tissue sarcomas. J Nucl Med 60(11):1537-1542

16. Hirsch FW, Sattler B, Sorge I et al (2013) PET/MR in children. Initial clinical experience in paediatric oncology using an integrated PET/MR scanner. Pediatr Radiol 43(7):860-875

17. Chawla SC, Federman N, Zhang D et al (2010) Estimated cumulative radiation dose from PET/CT in children with malignancies: a 5-year retrospective review. Pediatr Radiol 40(5):681-686

18. Spick C, Herrmann K, Czernin J (2016) 18F-FDG PET/CT and PET/MRI perform equally well in cancer: evidence from studies on more than 2,300 patients. J Nucl Med 57(3):420-430

19. Cheon GJ, Kim MS, Lee JA et al (2009) Prediction model of chemotherapy response in osteosarcoma by $18 \mathrm{~F}-\mathrm{FDG}$ PET and MRI. J Nucl Med 50(9):1435-1440

20. Rahbar K, Seifarth H, Schäfers M et al (2012) Differentiation of malignant and benign cardiac tumors using 18F-FDG PET/CT. J Nucl Med 53(6):856-863

21. Wagenknecht G, Kaiser HJ, Mottaghy FM, Herzog H (2013) MRI for attenuation correction in PET: methods and challenges. MAGMA 26(1):99-113

22. Bourcier K, Le Cesne A, Tselikas L et al (2019) Basic knowledge in soft tissue sarcoma. Cardiovasc Intervent Radiol 42(9):1255-1261

23. Sigal IR, Sebro R (2018) Preclinical PET tracers for the evaluation of sarcomas: understanding tumor biology. Am J Nucl Med Mol Imaging 8(6):428-440

24. Kratochwil C, Flechsig P, Lindner T et al (2019) ${ }^{68}$ Ga-FAPI PET/ CT: tracer uptake in 28 different kinds of cancer. J Nucl Med 60(6):801-805

Publisher's Note Springer Nature remains neutral with regard to jurisdictional claims in published maps and institutional affiliations. 